\title{
The new system of units
}

\author{
Joachim Fischer and Joachim Ullrich
}

\section{The redefinition of several physical base units planned for 2018 requires precise knowledge of the values of certain fundamental physical constants. Scientists are working hard to meet the deadlines for realizing the ultimate International System of Units.}

$\mathrm{T}$ he creation of the decimal metric system during the French Revolution and the subsequent deposition of two platinum standards representing the metre and the kilogram, on 22 June 1799 in the Archives de la République in Paris, was the first step in the development of the present International System of Units. Following the signing of the Convention of the Metre on 20 May 1875, new international prototypes for the metre and the kilogram were established in 1889 . These units, together with the astronomical second (based on the mean solar day) as the unit of time, constituted a mechanical unit system. Following the introduction of the ampere, the kelvin and the candela as the units for electric current, thermodynamic temperature and luminous intensity, respectively, the name Système International d'Unités, with the abbreviation SI, was given to the system in 1960. In 1971, the mole, the unit for amount of substance, completed the present SI.

Together with the derived units, the SI constitutes a coherent set of units (that is, without conversion factors) by which any measurable quantity of interest in research, industry, trade or society can be quantified. The signatory states of the Metre Convention represent about $98 \%$ of the world's economy, so the SI is the very basis of international trade, constituting a global measurement quality infrastructure through the national metrology institutes.

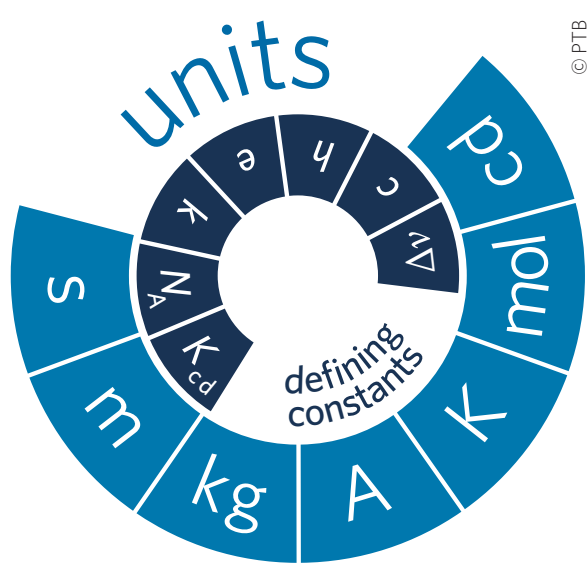

Figure 1 | Units and defining constants of the new SI. Note that there are no distinct one-to-one correspondences between units and constants (see also Table 1).

\section{Defining constants}

Extraordinary advances have been made in relating SI units to truly invariant quantities such as the fundamental constants of physics and the properties of atoms. Recognizing the importance of linking SI units to such invariant quantities, the 26th General Conference on Weights and Measures in 2018 will ratify a new definition of the SI based on the use of a set of seven such constants as references for the definitions. The value of any one of these seven constants

Table 1 | The seven defining constants of the new SI and the corresponding units they define.

\begin{tabular}{llll} 
Defining constant & Symbol & Numerical value & Unit \\
\hline Hyperfine splitting of caesium & $\Delta v\left({ }^{133} \mathrm{Cs}\right)_{\mathrm{hfs}}$ & $9,192,631,770$ & $\mathrm{~Hz}=\mathrm{s}^{-1}$ \\
Speed of light in vacuum & $c$ & $299,792,458$ & $\mathrm{~m} \mathrm{~s}^{-1}$ \\
Planck constant & $h$ & $6.626070040 \times 10^{-34}$ & $\mathrm{~J} \mathrm{~s}=\mathrm{kg} \mathrm{m}^{2} \mathrm{~s}^{-1}$ \\
Elementary charge & $\mathrm{e}$ & $1.6021766208 \times 10^{-19}$ & $\mathrm{C}=\mathrm{A} \mathrm{s}$ \\
Boltzmann constant & $k$ & $1.38064852 \times 10^{-23}$ & $\mathrm{~J} \mathrm{~K}^{-1}=\mathrm{kg} \mathrm{m}^{2} \mathrm{~s}^{-2} \mathrm{~K}^{-1}$ \\
Avogadro constant & $N_{\mathrm{A}}$ & $6.022140857 \times 10^{23}$ & $\mathrm{~mol}^{-1}$ \\
Luminous efficacy & $\mathrm{K}_{\mathrm{cd}}$ & 683 & $\mathrm{~cd} \mathrm{sr} \mathrm{W} \mathrm{W}^{-1}=\mathrm{cd} \mathrm{sr} \mathrm{kg}^{-1} \mathrm{~m}^{-2} \mathrm{~s}^{3}$
\end{tabular}

The numerical values are taken from the 2014 CODATA adjustment without the present associated uncertainties (not applicable to $\Delta v\left({ }^{133} \mathrm{Cs}\right)_{\mathrm{hts}}$ and $\left.\mathrm{c}\right)$ and may slightly change by 2018. is written as the product of a numerical coefficient and a unit, $Q=\{Q\}[Q]$, where $Q$ denotes the value of the constant and $\{Q\}$ its numerical value when expressed in the unit $[Q]$. By fixing the exact numerical value that is, not assigning any uncertainty to it the unit becomes defined, as the product of the numerical value and the unit must equal the value of the constant, which is invariant.

The so-called defining constants are the following: the frequency of the ground-state hyperfine splitting of the caesium-133 atom $\Delta v\left({ }^{133} \mathrm{Cs}\right)_{\mathrm{hfs}}$, the speed of light in vacuum $c$, the Planck constant $h$, the elementary charge $e$, the Boltzmann constant $k$, the Avogadro constant $N_{\mathrm{A}}$ and the luminous efficacy $K_{\mathrm{cd}}$ (see Table 1 and Fig. 1). Combinations of these constants define the units second $(\mathrm{s})$, metre (m), kilogram (kg), ampere (A), kelvin $(\mathrm{K})$, mole (mol) and candela $(\mathrm{cd})-$ and thus the entire SI.

One of the consequences of the redefinitions (Table 1) is that a distinction between (the former) base units and derived units is no longer necessary. As a second important feature, the definition and practical realization of the units will be decoupled in the new SI. Indeed, although the definitions of units may remain unchanged over a long period of time, their practical realizations can be established by many different experiments with ever-increasing accuracy - described in so-called mise-en-pratiques - thus allowing for experiments yet to be devised.

\section{Implications of the new definitions}

The second and the metre have already been successfully defined through fundamental constants. Fixing the numerical value of the hyperfine splitting of caesium defines the second as the duration of $9,192,631,770$ periods of the radiation that corresponds to the transition between the two hyperfine levels of the caesium atom. Having defined the second, fixing the numerical value of the speed of light means that the metre is the path length travelled by light in vacuum during a time interval of $1 / 299,792,458$ of a second. 
In 2018, the units kilogram, ampere, kelvin and mole will be defined in a similar way. The effect of fixing the numerical value of the Planck constant is a definition of the unit $\mathrm{kg} \mathrm{m}^{2} \mathrm{~s}^{-1}$ (the unit of the physical quantity called action). Together with the definitions of the second and the metre, this leads to a definition of the kilogram; macroscopic masses can be measured in terms of $h, \Delta v\left({ }^{133} \mathrm{Cs}\right)_{\mathrm{hfs}}$ and $c$. One way of establishing a mass scale is by counting the number of atoms in a silicon single-crystal sphere using the X-ray crystal density (XRCD) approach - probing the regular arrangement of atoms in a perfect lattice and multiplying it by the known mass of a silicon atom (the ${ }^{28} \mathrm{Si}$ isotope $)^{1}$. Another route to the kilogram is based on balancing electric and gravitational forces in a so-called watt balance $^{2}$ : in this scheme, the weight of a test mass is compared with the force generated by a coil, the electric power of which is measured very accurately by making use of the Josephson and quantum-Hall effects. The number chosen for the numerical value of the Planck constant will be such that at the time of adopting the definition, the kilogram is equal to the mass of the international prototype currently used for the definition of mass, within the uncertainty of the combined best estimates of the value of the Planck constant at that moment. These estimates are calculated regularly by the CODATA task group on fundamental constants ${ }^{3}$. (The mission of CODATA, the Committee on Data for Science and Technology, is to improve the quality, reliability, management and accessibility of data of importance to all fields of science and technology.) Subsequently, the mass of the international prototype will become a quantity to be determined experimentally.

The impact of fixing the numerical value of the elementary charge is that the ampere will become the electric current that corresponds to the flow of $1 /\left(1.6021766208 \times 10^{-19}\right)$ elementary charges per second. Electrical quantities (such as voltage, current and resistance) will be defined by fixing the value of $e$ (and $\left.\Delta v\left({ }^{133} \mathrm{Cs}\right)_{\mathrm{hfs}}\right)$ instead of the permeability of vacuum $\mu_{0}$ (which will have an uncertainty equal to that of the fine structure constant $\alpha=\mu_{0} e^{2} c / 2 h$ ). The conventional (defined) values of the Josephson and von Klitzing constants $K_{\mathrm{J}-90}$ and $R_{\mathrm{K}-90}$ will no longer be needed; these were introduced because they allowed a more precise realization of the electrical units than via today's definition of the ampere. In the future, the numerical values of the Josephson and von Klitzing constants $K_{\mathrm{J}}$ and $R_{\mathrm{K}}$ will be fixed in terms of the constants $e$ and $h$ (via the relationships $K_{\mathrm{J}}=2 e / h$ and $\left.R_{\mathrm{K}}=h / e^{2}\right)$. In particular, the volt and the ohm will be

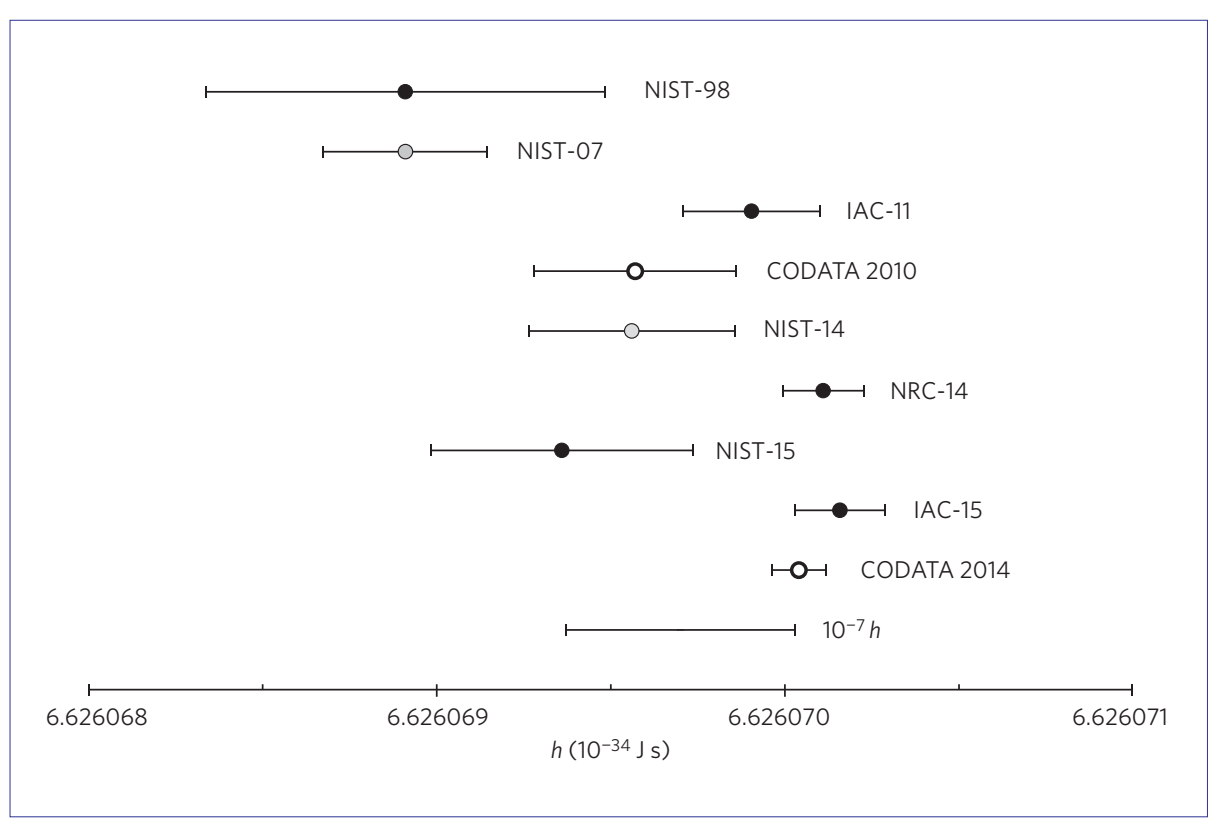

Figure 2 | All measurements that contributed (closed black data points) to the 2014 CODATA adjusted value of the Planck constant, along with the $2010^{3}$ and $2014^{18}$ CODATA values (open black data points), and the results NIST-07 and NIST-14 (grey data points) that were replaced by the value NIST-15. Values and standard uncertainties are taken from refs 10-16.

directly realizable, thus making the quantum realizations of the electrical units consistently embedded in the new SI. In fact, there will be a step change in the electrical units realized from quantum standards when the numerical values $K_{\mathrm{J}-90}$ and $R_{\mathrm{K}-90}$, which have been in use for more than 20 years, are abrogated in favour of the new fixed numerical values.

Fixing the numerical value of the Boltzmann constant means that the kelvin is equal to the change of thermodynamic temperature that results in a change of thermal energy $k T$ by $1.38064852 \times 10^{-23} \mathrm{~J}$. Today's definition of the kelvin is based on an exact temperature value assigned to the triple point of water ${ }^{4}$. After redefinition, this value will exhibit an uncertainty equal to that of the (currently experimentally determined) Boltzmann constant. With the new definition, it is evident that thermodynamic temperature can be realized directly at any point in the scale without referring to the singular temperature of the triple point of water. At present, thermometry relies on international temperature scales (ITSs), which were developed to give results that are in close agreement with the thermodynamic temperature, and are derived from a series of temperature fixed points (and interpolations between them) that are given conventional values approximating the corresponding thermodynamic temperatures ${ }^{5}$. Deviations between an ITS - the current ITS was agreed upon in 1990 - and the corresponding thermodynamic temperatures are made available to the user community by the Consultative Committee for Thermometry (CCT). It is expected that the new route with direct traceability to the SI will initially only be used in temperature ranges where primary thermometric methods offer lower uncertainties than ITS- $90^{6}$ (for example, below $20 \mathrm{~K}$ and above $1,300 \mathrm{~K}$ ).

Fixing the numerical value of the Avogadro constant means that the mole is the amount of substance of a system that contains $6.022140857 \times 10^{23}$ specified elementary entities. The value for $N_{\mathrm{A}}$ will be based on the result of the XRCD experiment. The current definition of the mole defines the value of the molar mass of carbon- $12, M\left({ }^{12} \mathrm{C}\right)$, as exactly $0.012 \mathrm{~kg} \mathrm{~mol}^{-1}$. In the future, $M\left({ }^{12} \mathrm{C}\right)$ will no longer be exactly defined but will have an associated uncertainty equal to that of the molar Planck constant $N_{\mathrm{A}} h$, which is far smaller than that achieved in any practical chemical measurement ${ }^{7}$.

\section{Requirements for the defining constants}

To guarantee minimal changes when adopting the new definitions, the Consultative Committee for Mass and Related Quantities (CCM) passed a recommendation ${ }^{8}$ based on a review published in $2010^{9}$ to impose quantitative requirements on the results of determinations of $h$. Among others, the following essential conditions must be met: (1) at least three independent experiments, including watt balance and XRCD experiments, should 


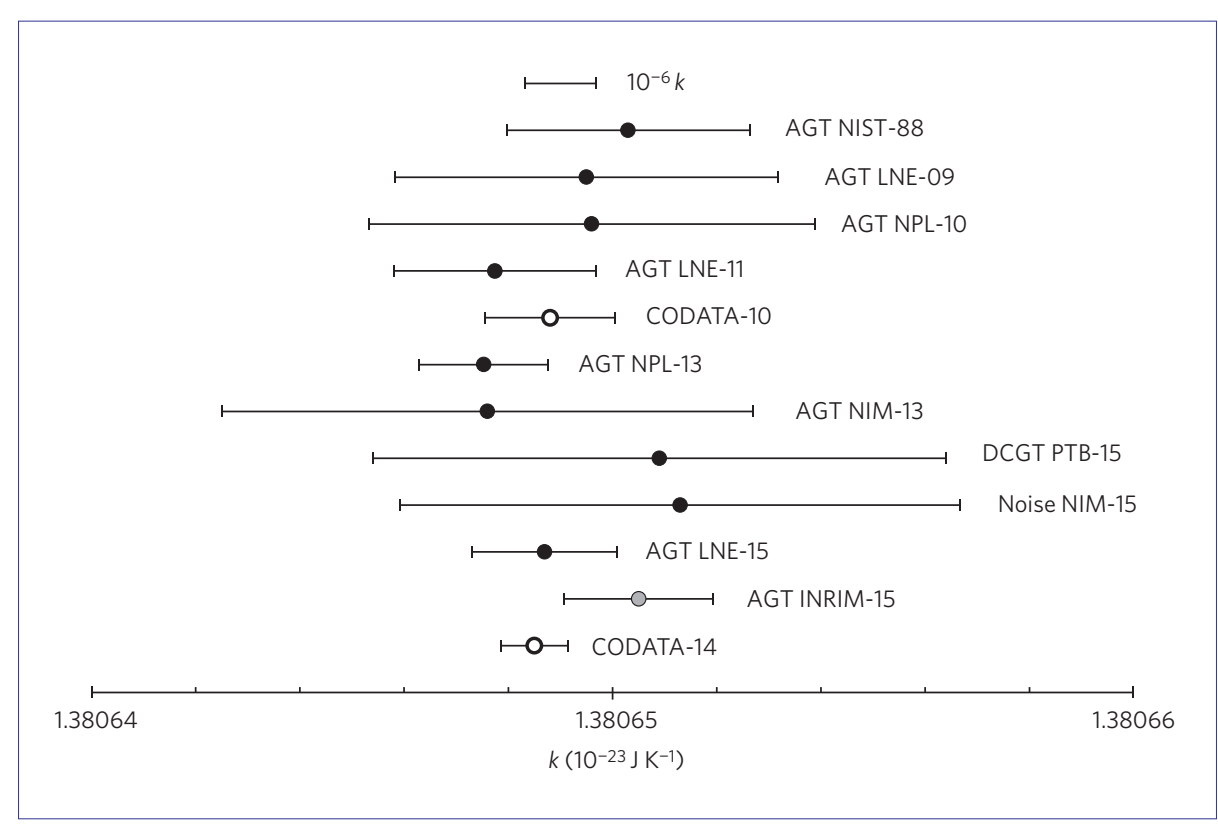

Figure 3 | All measurements that contributed (closed black data points) to the 2014 CODATA adjusted value of the Boltzmann constant, along with the $2010^{3}$ and $2014^{18}$ CODATA values (open black data points), and the acoustic-gas-thermometry result (grey data point) obtained at INRIM (Istituto Nazionale di Ricerca Metrologica, Italy) but published after the deadline for the 2014 adjustment. Values and standard uncertainties are taken from refs 19-25.

yield consistent values of the Planck constant with relative standard uncertainties not larger than 5 parts in $10^{8}$; and (2) at least one of these results should have a relative standard uncertainty not larger than 2 parts in $10^{8}$. Here and in the following all uncertainty values given are relative standard uncertainties.

At the time of publication of the 2010 CODATA evaluation of the fundamental constants, there was a significant discrepancy between the available experimental values for the Planck constant (Fig. 2) ${ }^{3}$. This concerned values (NIST-98 and NIST-07) obtained at NIST (National Institute of Standards and Technology, USA) with different watt balance experiments ${ }^{10,11}$ and a value (IAC-11) of the International Avogadro Collaboration based on the XRCD method ${ }^{12}$.

An extensive review of the NIST watt balance data led to a revised value of the Planck constant published in $2014^{13}$ with an increased uncertainty (compared with NIST-07) of 4.5 parts in $10^{8}$. The result from NRC (National Research Council, Canada), also published in $2014^{14}$, has an uncertainty of only 1.8 parts in $10^{8}$, which meets the second CCM condition. Most recently, NIST researchers have reported a single value (NIST-15) for $h$ with an uncertainty of 5.6 parts in $10^{8}$ based on all the data obtained using their current watt balance ${ }^{15}$, thereby replacing their values reported in 2007 and
2014 (marked by grey dots in Fig. 2). The value obtained with an earlier apparatus (NIST-98) stands as an independent result.

In 2015 the IAC collaboration reported a new value (IAC-15) with an uncertainty of 2.0 parts in $10^{8}$, which also fulfils the second CCM condition ${ }^{16}$. Moreover, IAC researchers successfully addressed issues concerning the correlation between the 2011 and 2015 IAC values, thereby allowing both sets of data to be used for the 2014 adjustment. All this led to an uncertainty of only 1.2 parts in $10^{8}$ for the 2014 adjusted value of $h$.

The CCT similarly imposed requirements on the value of the Boltzmann constant. They recommended two conditions ${ }^{17}$ that are to be met before the new definition can be adopted: (1) the uncertainty of the adjusted value of $k$ should be less than 1 part in $10^{6}$; and (2) the determination of $k$ should be based on at least two fundamentally different methods, of which at least one result for each must have an uncertainty of less than 3 parts in $10^{6}$.

Measured values of $k$ taken into account in the 2014 CODATA adjustment ${ }^{18}$ are shown in Fig. 3. The 2010 CODATA adjustment ${ }^{3}$ had an uncertainty of 9.1 parts in $10^{7}$, which fulfils the first condition of the CCT. The second condition was not met at all, as only experiments at NIST, LNE (Laboratoire National de Métrologie et d'Essais, France) and NPL (National Physical
Laboratory, UK) - all based on acoustic gas thermometry - achieved uncertainties below 3 parts in $10^{6}$ (ref. 19). Based on these results, and thanks to the resolving of a discrepancy between the values of LNE-11 and NPL- $13^{20}$, the uncertainty of the 2014 adjusted value of $k$ has been significantly reduced to 5.7 parts in $10^{7}$. Experiments using dielectric-constant gas thermometry developed at PTB (PhysikalischTechnische Bundesanstalt, Germany) ${ }^{21}$ and noise thermometry at NIM (National Institute of Metrology, China) $)^{22}$ achieved uncertainties close to 4 parts in $10^{6}$. These two experiments are certainly candidates for meeting the second CCT requirement ${ }^{23}$.

\section{Summary}

Encouraging progress has been made in lowering the uncertainties of $N_{\mathrm{A}}, h$ and $k$ to the extent that the conditions for redefinition are either met or within reach. Moreover, new results for $h$ and $N_{\mathrm{A}}$ are expected to be published by NIST and the IAC collaboration, and for $k$ by PTB and NIM, before the deadline (1 July 2017) set by the Consultative Committee for Units (CCU) for data that will be used to fix the numerical values of the constants for the SI redefinitions. A number of coordination and communication initiatives have been launched, such as a CCM-CCU roadmap for the kilogram ${ }^{26}$ and a new-SI awareness campaign initiated by the CIPM (Comité International des Poids et Mesures, France).

Redefining the SI units by fixing the numerical values of natural constants - as suggested by Max Planck in $1900^{27}$ — will have far-reaching benefits for innovations in industry, serving society and fostering science and research. In Planck's visionary words, these units will "necessarily retain their validity for all times and cultures, even extra-terrestrial and nonhuman," meaning that they are stable and realizable everywhere. New primary methods are emerging for measuring, for example, masses at the milligram level or temperature over extended scales, and most importantly, uncertainties in electrical metrology will be significantly reduced. There is no doubt that these redefinitions will open the door to continuous technical developments towards ever-decreasing uncertainties in the realization of the units, without the need for further redefinition.

Joachim Fischer and Joachim Ullrich are at the Physikalisch-Technische Bundesanstalt, Abbestrasse 2-12, 10587 Berlin, Germany, and at the Physikalisch-Technische Bundesanstalt, Bundesallee 100, 38116 Braunschweig, Germany. e-mail: joachim.fischer@ptb.de; joachim.ullrich@ptb.de 
References

1. Bettin, H. et al. Ann. Phys.-Berlin 525, 680-687 (2013).

2. Steiner R. et al. IEEE T. Instrum. Meas. 56, 592-596 (2007).

3. Mohr, P. J. et al. Rev. Mod. Phys. 84, 1527-1605 (2012).

4. de Podesta, M. Nature Phys. 12, 104 (2016).

Moldover, M. R., Tew, W. L. \& Yoon, H. W. Nature Phys. 12, 7-11 (2016).

6. Fischer, J. et al. Int. J. Thermophys. 28, 1753-1765 (2007).

Milton, M. et al. Metrologia 51, R21-R30 (2014).

8. Consultative Committee for Mass and Related Quantities (CCM): Report of the 12th Meeting, Recommendation G 1 (BIPM, 2010); http://go.nature.com/vy4ADR
9. Gläser, M. et al. Metrologia 47, 419-428 (2010).

10. Williams, E. R. et al. Phys. Rev. Lett. 81, 2404-2407 (1998).

11. Steiner, R. Rep. Prog. Phys. 76, 016101 (2013).

12. Andreas, B. et al. Phys. Rev. Lett. 106, 030801 (2011).

13. Schlamminger, S. et al. Metrologia 51, S15-S24 (2014).

14. Sanchez, C. et al. Metrologia 51, S5-S14 (2014).

15. Schlamminger, S. et al. Metrologia 52, L5-L8 (2015).

16. Azuma, Y. et al. Metrologia 52, 360-375 (2015).

17. Recommendations of the Consultative Committee for

Thermometry Submitted to the International Committee for

Weights and Measures: Recommendation $T 1$ (BIPM, 2014);

http://go.nature.com/lz7ibZ

\author{
18. Mohr, J. et al. Preprint at http://arxiv.org/abs/ \\ 1507.07956 (2015) \\ 19. Moldover, M. R. et al. Metrologia 51, R1-R19 (2014). \\ 20. de Podesta, M. et al. Metrologia 52, S353-S363 (2015). \\ 21. Gaiser, C. et al. Metrologia 52, S217-S226 (2015). \\ 22. Qu, J. et al. Metrologia 52, S242-S256 (2015). \\ 23. Fischer, J. Metrologia 52, S364-S375 (2015). \\ 24. Pitre, L. et al. Metrologia 52, S263-S273 (2015) \\ 25. Gavioso, R. M. et al. Metrologia 52, S274-S304 (2015). \\ 26. Joint CCM and CCU Roadmap towards the Redefinition of the SI \\ in 2018 (BIPM, 2015); http://go.nature.com/MYwKRV \\ 27. Planck, M. Ann. Physik 1, 69-122 (1900).
}

\title{
Advances in thermometry
}

\author{
Michael R. Moldover, Weston L. Tew and Howard W. Yoon
}

\section{The past 25years have seen tremendous progress in thermometry across the moderate temperature range of $1 \mathrm{~K}$ to $1,235 \mathrm{~K}$. Various primary thermometers, based on a wide range of different physics, have uncovered errors in the International Temperature Scale of 1990, and set the stage for the planned redefinition of the kelvin.}

$\mathrm{T}$ hermodynamic temperature $T$ is the physical property that two objects have in common when they are isolated from the rest of the Universe and are allowed to exchange energy with each other. The actual shared property is the average energy per degree of freedom. In the International System of Units (SI), the unit of energy is the joule, and 'number of degrees of freedom' is a dimensionless number. Therefore, the property that objects in thermodynamic equilibrium share can be expressed in joules per degree of freedom. For example, if we allow the air in a comfortable room to come into equilibrium with a thermometer, the thermometer will read $4.04 \times 10^{-21} \mathrm{~J}$ per degree of freedom. For historical reasons, temperature is expressed in kelvin, therefore the aforementioned thermometer reading in kelvin is $T=293 \mathrm{~K}$. The conversion factor between temperature expressed in kelvin and joules per degree of freedom is the Boltzmann constant, $k_{\mathrm{B}}=1.38064852 \times 10^{-23} \mathrm{~J} \mathrm{~K}^{-1}$. It is important to keep in mind that the product $k_{\mathrm{B}} T$ is the physical quantity that characterizes the thermal equilibrium between a particular pair of objects, one of which might be a thermometer.

\section{The (re)definition of the kelvin}

Today, the kelvin, the unit of thermodynamic temperature, is "the fraction $1 / 273.16$ of the thermodynamic temperature of the triple point of water". (Here, we abbreviate "the temperature of the triple point of water" as $T_{\text {TPW. }}$.) As of 2014, $k_{\mathrm{B}}$ has been measured with a relative standard uncertainty of $u_{\mathrm{r}}\left(k_{\mathrm{B}}\right)=5.7 \times 10^{-7}$ and, by definition, $u_{\mathrm{r}}\left(T_{\mathrm{TPW}}\right)=0$; therefore, $u_{\mathrm{r}}\left(k_{\mathrm{B}} T_{\mathrm{TPW}}\right)=5.7 \times 10^{-7}$ (ref. 2). The present SI definition of the kelvin has two disadvantages that will disappear when $k_{\mathrm{B}}$ is assigned a fixed value ${ }^{3}$ and the relative uncertainty of $k_{\mathrm{B}} T_{\mathrm{TPW}}$ is entirely due to that of $T_{\mathrm{TPW}}$. First, the special role of the triple point of water in defining $T$ will be eliminated; however, the measured value of $T_{\text {TPW }}$ will still be close to $273.16 \mathrm{~K}$. Although this change is conceptually important, triple-point-of-water cells will continue to be essential for practical thermometry because they are inexpensive, stable, portable and highly reproducible. Second, with the present definition of the kelvin, no matter how accurately $k_{\mathrm{B}} T$ is measured, the resulting relative uncertainty of the temperature $u_{\mathrm{r}}(T)$ must de facto be greater than or equal to the uncertainty of $k_{\mathrm{B}}$. After the planned redefinition of the kelvin, it will be possible, in principle, for a future metrologist to measure $k_{\mathrm{B}} T_{x}$ accurately at a temperature $T_{x}$ far from $T_{\mathrm{TPW}}$ (say, $T_{x}=2,000 \mathrm{~K}$ ) with an uncertainty attributed to $T_{x}$ that is smaller than the uncertainty of $T_{\text {TPW. }}$. (After $k_{\mathrm{B}}$ is fixed in 2018, this possibility will be realized only in the unlikely circumstance that $u_{\mathrm{r}}\left(T_{x}\right) \leq u_{\mathrm{r}}\left(T_{\mathrm{TPW}}\right)=5.7 \times 10^{-7}$.

\section{Thermodynamic thermometry}

To accurately measure $k_{\mathrm{B}} T$ (the average energy per degree of freedom), a metrologist must use a primary thermometer; that is, any instrument that has a well-understood connection between a measurable quantity and $k_{\mathrm{B}} T$. In the following, we briefly discuss five different measurable quantities that are used or can be used for primary thermometry: (1) the pressure of a gas in a constant volume; (2) the speed of sound in a monatomic gas; (3) the dielectric constant of a gas; (4) the radiation emitted by a black body; and (5) the power spectral density of Johnson-noise in a resistor. References and more detailed discussions can be found in reviews by Fischer and colleagues ${ }^{4,5}$.

\section{Gas thermometry}

Gas thermometry relies on the statisticalmechanical connection between the three kinetic degrees of freedom of an ideal gas and thermodynamic temperature, giving $(1 / 2) \mathrm{m}\left\langle v^{2}\right\rangle=3 k_{\mathrm{B}} T$, where $m$ is the mass of an atom and $\left\langle v^{2}\right\rangle$ is the mean-square velocity of the atom. An immediate consequence of this connection is the ideal gas law, which relates the number density $n$ and pressure $p$ to temperature, such that $k_{\mathrm{B}} T=p / n$. For monatomic gases, kinetic theory also predicts $\left\langle v^{2}\right\rangle=9 u^{2} / 5$, where $u$ is the speed of sound in the gas. The speed of sound is therefore connected to temperature by $k_{\mathrm{B}} T=3 m u^{2} / 5$.

Prior to 1990, many of the measurements of thermodynamic temperature $T$ conducted at national metrology institutes (NMIs) used helium-filled, constant-volume gas thermometers. Such thermometers involved a fixed quantity of helium confined within a cavity known as the 'thermometer bulb. The pressure of the helium gas was measured as the temperature was changed from the defining temperature $T_{\text {TPW }}$ to an unknown temperature $T_{x}$. Because helium is not an ideal gas, the ratio of the pressures yielded an approximation to the ratio of the temperatures, giving $p\left(T_{x}\right) / p\left(T_{\mathrm{TPW}}\right) \approx T_{\mathrm{x}} / T_{\mathrm{TPW}}$, which becomes exact in the limit of zero pressure. The pressure ratio measurements 